\title{
Effect of Graphene Nanoplates on Phase Structure and Electrical Properties of Epoxy/Polyetherimide Composite
}

\author{
Yong-Ling PAN ${ }^{1}$, Chuan-Guo MA ${ }^{1,2, \text { a }}{ }^{*}$ Hua-Mei WAN ${ }^{1}$, Ping-Ying TAO ${ }^{1}$, Qi \\ $\mathrm{SHI}^{1}$, De-Shui HUANG ${ }^{1}$, and Ji-Xing WANG ${ }^{1}$ \\ ${ }^{1}$ School of Material Science and Engineering, Guilin University of Electronic Technoloy, Guilin \\ 541004, China \\ ${ }^{2}$ Guangxi Key laboratory of information materials Guilin 541004 China, Guilin, 541004, China \\ amachuanguo@guet.edu.cn \\ ${ }^{*}$ Corresponding author
}

Keywords: Graphene nanoplates, Epoxy resin, Polyetherimide, Phase structure, Electrical properties.

\begin{abstract}
Epoxy resin/polyetherimide/graphene nanoplates (EP/PEI/GnPs) composites were prepared by solution blending, and the effects of GnPs on the phase structure and electrical properties of the systems were investigated by scanning electron microscopy (SEM), optical microscopy (OM), digital ultrahigh resistance and microcurrent measuring meter and precision impedance analyzer. The results show that the phase separation morphology of the composites change from sea-island structure to bicontinuous structure and phase inversion structure with the increase of GnPs content. The composites have an electrical percolation threshold of $0.85 \mathrm{wt} \%$. A high dielectric constant of more than 310 at $1 \mathrm{kHz}$ could be obtained for the composite with a filler content of $1.75 \mathrm{wt} \%$.
\end{abstract}

\section{Introduction}

Conductive polymer composites (CPCs) are widely used as anti-static materials, conductive materials, electromagnetic shielding materials (EMI), semiconductors materials, sensors and electric materials due to their tunable properties and ease of fabrication [1-3]. When conductive filler is introduced into polymer matrix the resistivity of system will be reduced drastically as conductive filler content increased to a certain critical value, which is called percolation threshold. The increase of percolation threshold means more conductive filler is required to achieve the ideal electrical performance, which may increase the difficulty of processing, worsen the mechanical properties and also raise the price of composites. Therefore, many researches have being focused on the problem how to reduce the percolation threshold while insuring the electrical properties have not been reduced. And regulating the structure of composites is one of the effective methods to reduce the percolation threshold.

Recently, carbon filler such as carbon black (CB), carbon nanotubes (CNTs) and graphene oxide have been introduced into epoxy resin/polyetherimide (EP/PEI) binary systems to control the phase structure of composites. Ma et al. [4] reported a novel double-percolation structural conductive polymer composite based on EP/PEI/CB. Zhang et al. [5] found that the introduction of CNTs increased the viscosity of the EP/PEI systems, causing an inhibiting effect on phase separation. Wu et al. [6] reported that the phase separation was suppressed by graphene oxide (GO) in the EP/PEI systems. To the best of our knowledge, the conductive graphene haven't been applied to an EP/PEI binary system. In this work, graphene nanoplates ( $\mathrm{GnPs}$ ) was incorporated into EP/PEI binary system, and the effects of GnPs on phase structure, volume resistivity and dielectric properties of epoxy/polyetherimide blend system were studied. 


\section{Experimental}

\section{Materials}

Diglycidyl ether of bisphenol A resin (DGEBA, E51) was purchased from China Petroleum \& Chemical Corporation (China). PEI (Ultem-1000) was provided by GE Co., Ltd (USA). The 4, 4'-diaminodiphenyl sulfone (DDS) used as curing agent was produced by Zhejiang Dragon Chemical Group Co., Ltd (China). Graphene nanoplates (GnPs) with a thickness of 3-10 nm was produced by Nanjing XFNANO Materials Tech Co., Ltd. Polyvinylpyrrolidone (PVP, K60) was bought from Najing Shuguang Chemical Group Co., Ltd (China). Dichloromethane (DCM) was purchased from Xilong Chemical Co., Ltd (China). All materials and reagents were used as received.

\section{Preparation of Composites}

The same amount of GnPs and PVP were dispersed in $50 \mathrm{ml}$ DCM, and a homogenous mixture was obtained by employing an ultrasonic probe sonicator at $450 \mathrm{~W}$ for $30 \mathrm{~min}$. And the above mixture was sonicated for another $30 \mathrm{~min}$ after PEI/DCM solution containing $1 \mathrm{~g}$ PEI was added. Then the mixture was added to $4 \mathrm{~g}$ preheated EP at $100^{\circ} \mathrm{C}$ under continuously stirring. After most of DCM was removed, $1.32 \mathrm{~g}$ DDS was added and stirred for $5-6 \mathrm{~min}$ at $130{ }^{\circ} \mathrm{C}$ until DDS was fully dissolved. Finally, the sample was casted into a standard mold and placed in a vacuum oven to remove the residual solvent. The curing cycle was $4 \mathrm{~h}$ at $170^{\circ} \mathrm{C}$.

\section{Characterization}

Scanning electron microscopy (SEM) (Quanta FEG 450, USA) was used to analyze the phase structures of the composites and the distributions of GnPs. The volume resistance $\left(R_{V}\right)$ of the composites was measured by a high resistance meter (EST121, China) according to ASTM D257. Precision impedance analyzer (Agilent 4294A, USA) was used to collect the dielectric data in the frequency range of $1 \mathrm{kHz}-110 \mathrm{MHz}$ at room temperature.

\section{Results and Discussion}

\section{Morphological Study by SEM}

Three different types of phase morphologies were observed in EP/PEI/GnPs ternary composites with different GnPs content in Fig.1. The EP/PEI binary composite shows sea-island morphology (Fig. 1(a)) in which the fine PEI globules with a size about $1 \mu \mathrm{m}$ are uniformly distributed in EP-rich phase. And the phase structure is still sea-island morphology (Fig. 1(b)) after $0.1 \mathrm{wt} \%$ GnPs was added to the system. As illustrated in Fig. 1(c), the EP/PEI/GnPs composite with a GnPs loading of 0.75wt\% shows co-continuous morphology, and the GnPs (arrow) are selectively distributed in PEI-rich phase. While the composite shows phase-inverted structure (Fig. 1(d)) when GnPs content is increased to 2 wt\%, and the severely agglomerated GnPs was observed.

In the system of EP modified with PEI the morphologies of cured samples can be controlled by changing the relative rate of curing reaction and phase separation [5], which is influenced by the introduction of the third component GnPs, resulting in the changing of phase structure from sea-island morphology to co-continuous and phase-inverted morphology. 

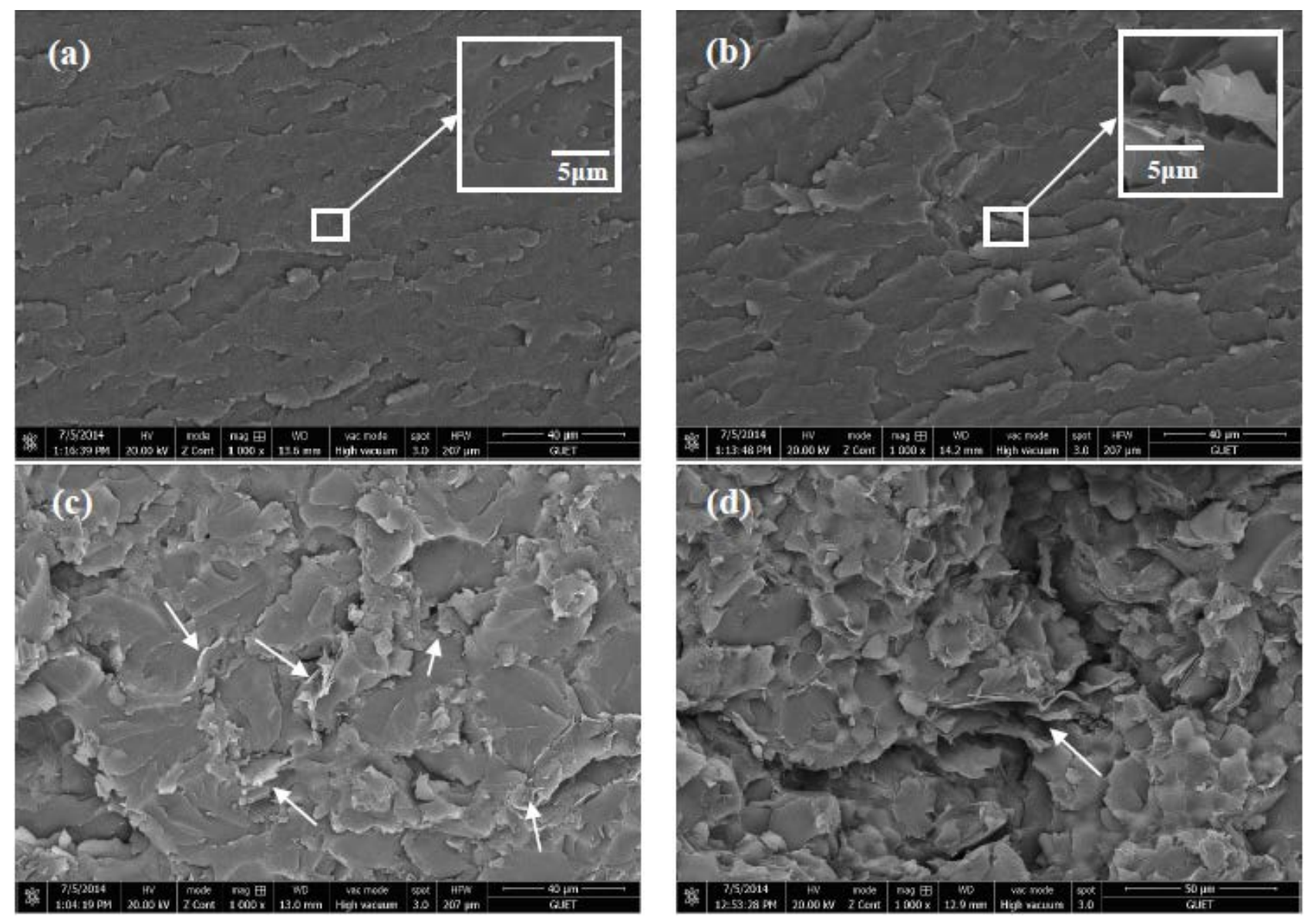

Fig.1 SEM micrographs of the fractured surface of composites with different GnPs content.

(a)without GnPs (b) $0.1 w t \%$ (c) $0.75 w t \%$ (d) $2 w t \%$

\section{Electrical Properties of Composites}

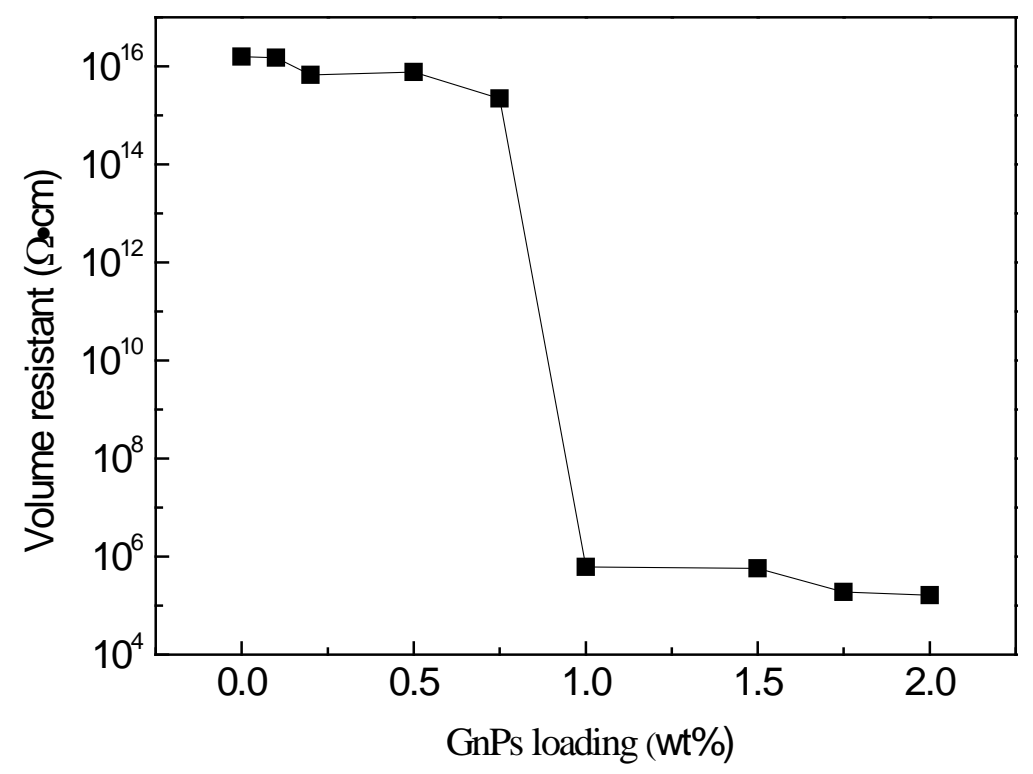

Fig.2. Volume resistivity of the composites as a function of GnPs loading.

Fig.2 shows the volume resistivity of EP/PEI blend and EP/PEI/GnPs ternary composites. As compared with that of the EP/PEI blend, a slight decrease in volume resistivity is observed in the ternary composites with a GnPs loading of $0.75 \mathrm{wt} \%$ and the ternary composites are still insulating. Because the conductive GnPs particles are separated from each other when the concentrations of 
GnPs are below $0.75 \mathrm{wt} \%$, thus the electrical properties of the composites are dominated by the matrix. While the volume resistivity drops dramatically from $2.18 \times 10^{15} \Omega \cdot \mathrm{cm}$ to $6.13 \times 10^{5} \Omega \cdot \mathrm{cm}$ as the GnPs filler increases from $0.75 \mathrm{wt} \%$ to $1 \mathrm{wt} \%$ and decreases a little to $5.7 \times 10^{5} \Omega \cdot \mathrm{cm}$ when the GnPs content increases to $1.5 \mathrm{wt} \%$, indicating that the electrical percolation falls between $0.75 \mathrm{wt} \%$ and $1 \mathrm{wt} \%$. The perfect conductive cross-linked network is formed through the interconnection of GnPs when the GnPs loading is $1 \mathrm{wt} \%$, so the further increase of GnPs content has little effect on the improvement of conductivity.

\section{Dielectric Properties of Composites}

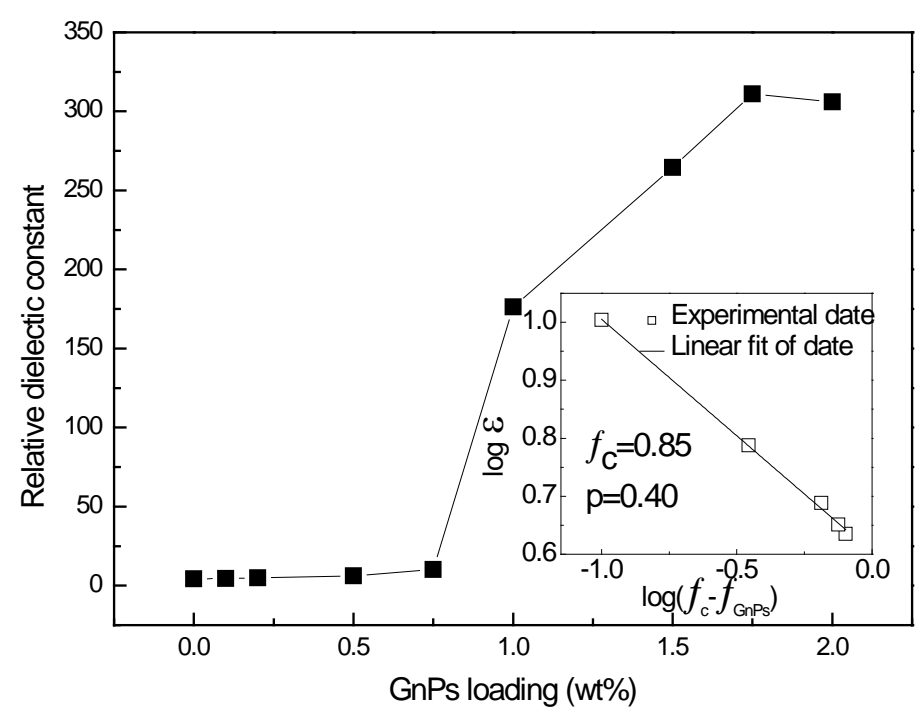

Fig.3. Relative dielectric constant of the composites as a function of GnPs content at $1 \mathrm{kHz}$ and room temperature. The inset shows the best fit of dielectric constant of the composites to equation (1).

Fig. 3 shows dielectric constant of the composites at $1 \mathrm{kHz}$. It is observed that the dielectric constant is significantly improved due to the introduction of GnPs. The dielectric constant increases relatively moderate when $\mathrm{f}_{\mathrm{GnPs}}<0.75 \mathrm{wt} \%$, indicating that there is no plentiful accumulation of interfacial charges inside the EP/PEI/GnPs composites. The dielectric constant increases rapidly after $0.75 \mathrm{wt} \%$, and that of the composite when $f_{\mathrm{GnPs}}=1.75 \mathrm{wt} \%$ is 311 , which is over 100 times higher than that of EP/PEI blend (about 3). When the GnPs content is near the percolation threshold, the dielectric constant can be expressed by the percolation-theory power law $[7,8]$,

$$
\varepsilon\left(f_{\text {GnPs }}\right) \propto\left(f_{c}-f_{G n P s}\right)^{-p} \text {, for } f_{G n P s}<f_{c} .
$$

Where $f_{\mathrm{c}}$ is the percolation threshold, $f_{\mathrm{GnPs}}$ is the weight percentage of $\mathrm{GnPs}, \varepsilon\left(f_{\mathrm{GnPs}}\right)$ is dielectric permittivity of the composites and $p$ is critical exponent. From the best fitting, we got $f_{c}=0.85 w t \%$ and $\mathrm{p}=0.40$. The critical exponent in the insulation region, $\mathrm{p}=0.40$, is smaller than the universal ones. The non-universal percolation exponent was reported by Chiteme et al. [9]. The spatial distribution and interaction of GnPs in polymer matrices, and the nature of the interparticle contacts, might be major factors in determining the critical exponents.

The dependence of dielectric properties with increased frequency ranging from $10^{3}$ to $10^{8} \mathrm{~Hz}$ for EP/PEI blend and EP/PEI/GnPs composites are illustrated in Fig.4. At a lower frequency range $\left(10^{3}-10^{4} \mathrm{~Hz}\right)$, the dielectric constant of EP/PEI/GnPs composites was improved dramatically when GnPs contents are above percolation threshold (0.85 wt\%), and up to higher than 230 when the filler content was above $1 \mathrm{wt} \%$. The dielectric constant decrease sharply in the frequency range of $10^{4}-10^{7}$ $\mathrm{Hz}$ because of polarization relaxation. According to the percolation theory, both the dielectric constant and dielectric loss exhibit a transition behavior in the vicinity of percolation threshold. Thus, relatively higher dielectric loss of the composites with a GnPs content over percolation threshold could be observed in Fig. 4(b). And the dielectric loss peaks are found around $10^{5} \mathrm{~Hz}$ and $10^{6} \mathrm{~Hz}$ for 
the composites with different GnPs content that are corresponding to the sharp decline of dielectric constant in Fig.4 (a), which is caused by polarization relaxation. At a lower frequency range $\left(10^{3}-10^{4}\right.$ $\mathrm{Hz}$ ), the dielectric constant of EP/PEI/GnPs composites was and up to higher than 230 when the filler content was above $1 \mathrm{wt} \%$ when GnPs contents are above percolation threshold.
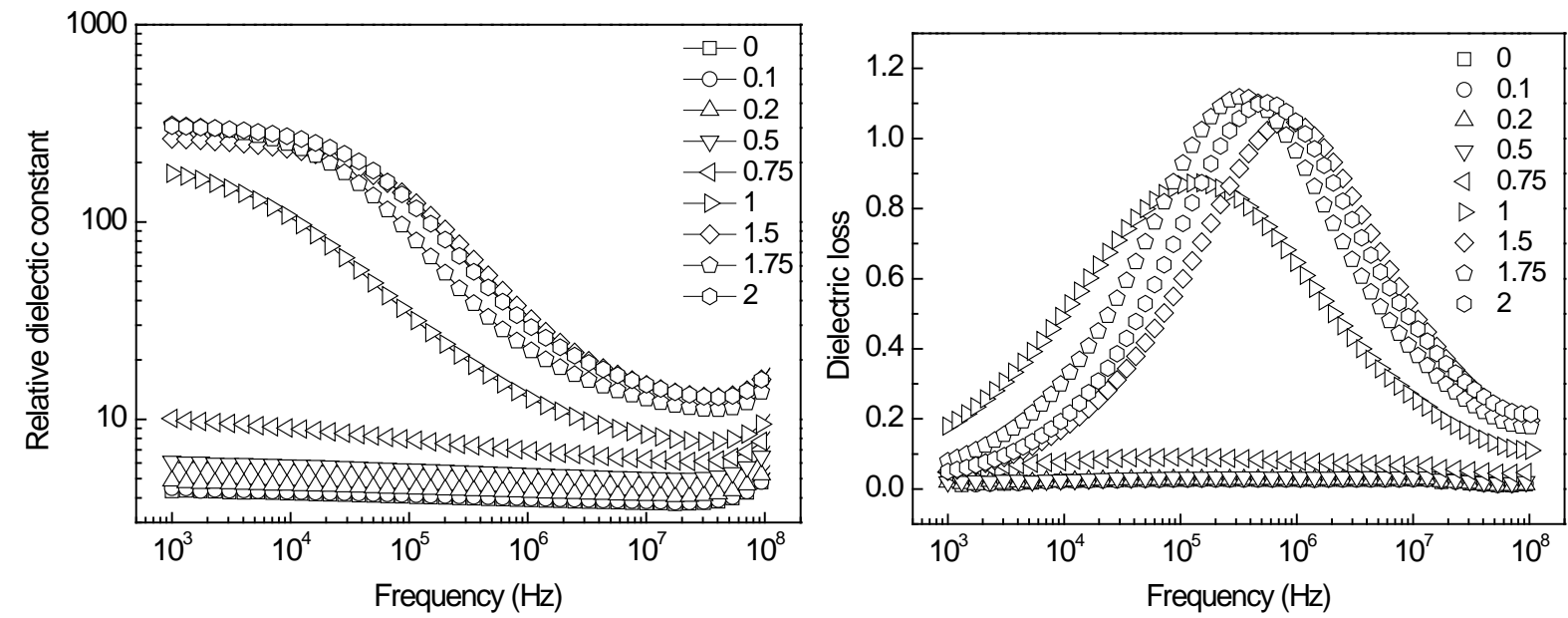

Fig.4. Dependence of relative dielectric constant (a) and dielectric loss (b) of composites with different GnPs content on frequency ranging from $1 \mathrm{kHz}$ to $110 \mathrm{MHz}$.

\section{Summary}

The phase structure of EP/PEI composite was effectively controlled by the introduction of the third component GnPs, and it changed from sea-island morphology to co-continuous and phase-inverted morphology. The EP/PEI/GnPs ternary composites had an electrical percolation threshold of 0.85 wt $\%$. The volume resistivity of composites decreased from $2.18 \times 10^{15} \Omega \cdot \mathrm{cm}$ to $6.13 \times 10^{5} \Omega \cdot \mathrm{cm}$ as the GnPs filler increases from $0.75 \mathrm{wt} \%$ to $1 \mathrm{wt} \%$. The dielectric constant of the composite with GnPs content of $1.75 \mathrm{wt} \%$ is the highest (about 311, at $\mathrm{l} \mathrm{kHz}$ ), which is over 100 times higher than that of EP/PEI blend (about 3).

\section{Acknowledgements}

The authors gratefully acknowledge the supports from the National Natural Science Foundation of China (Grant \#51363003) and National Undergraduate Training Program for Innovation and Entrepreneurship (201410595032).

\section{References}

[1] R.A. Antunes, M.C.L. De Oliveira, G. Ett, Carbon materials in composite bipolar plates for polymer electrolyte membrane fuel cells: a review of the main challenges to improve electrical performance, J. Power Sources. 196 (2011) 2945-2961.

[2] P.C. Ma, N.A. Siddiqui, G. Marom, Dispersion and functionalization of carbon nanotubes for polymer-based nanocomposites: a review, Composites Part A. 41 (2010) 1345-1367.

[3] T. Villmow, S. Pegel, A. John, Liquid sensing: smart polymer/CNT composites, Mater. Today. 14 (2011) 340-345.

[4] C.G. Ma, D.Y. Xi, M. Liu, Epoxy resin/polyetherimide/carbon black conductive polymer composites with a double percolation structure by reaction-induced phase separation, J. Compos. Mater. 47 (2013) 1153-1160. 
[5] J. Zhang, X.M. Xie, Effects of MWCNTs on phase separation in epoxy Resin/PEI blend systems, Acta Polym. Sin. 2 (2011) 215-222. (In Chinese)

[6] G. Yu, P.Y. Wu, Effect of chemically modified graphene oxide on the phase separation behaviour and properties of an epoxy/polyetherimide binary system, Polym. Chem. 5 (2014) 96-104.

[7] P. Fan, L. Wang, J. Yang, Graphene/poly (vinylidene fluoride) composites with high dielectric constant and low percolation threshold, Nanotechnology, 23 (2012) 365702.

[8] F. He, S. Lau, H.L. Chan, High dielectric permittivity and low percolation threshold in nanocomposites based on poly (vinylidene fluoride) and exfoliated graphite nanoplates, Adv. Mater. 21 (2009) 710-715.

[9] C. Chiteme, D.S. Mclachlan, Measurements of universal and non-universal percolation exponents in macroscopically similar systems, Physica B. 279 (2000) 69-71. 